\title{
Heart failure with preserved ejection fraction
}

\author{
Authors: Andrew R Harper, ${ }^{A}$ Hitesh C Patel ${ }^{B}$ and Alexander R Lyon ${ }^{C}$
}

Heart failure with preserved ejection fraction (HFpEF) represents a heterogeneous collection of conditions that are unified by the presence of a left ventricular ejection fraction $\geq 50 \%$, evidence of impaired diastolic function and elevated natriuretic peptide levels, all within the context of typical heart failure signs and symptoms. However, while HFpEF is steadily becoming the predominant form of heart failure, disease-modifying treatment options for this population remain sparse. This review provides an overview of the diagnosis, management and prevention of HFpEF for general physicians.

KEYWORDS: Heart failure, ejection fraction

\section{Introduction}

Heart failure (HF) is an important clinical syndrome affecting

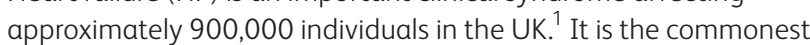
cause of hospitalisation in individuals above the age of 65 years and contributes to one in nine deaths. Patients with this diagnosis have a reduction in both quality of life and life expectancy (50\% of patients with the diagnosis are not expected to survive more than 5 years). ${ }^{2} \mathrm{HF}$ is a heterogeneous condition and is categorised into three groups based on ejection fraction (EF):

1 those with $\mathrm{HF}$ and a reduced EF (HFrEF where left ventricular EF [LVEF] is $<40 \%$ )

2 those with HF and a mid-range EF (HFmrEF where LVEF is 40-50\%)

3 those with HF and a preserved or normal EF (HFpEF where LVEF is $\geq 50 \%$ ).

There are limited treatment options available to either improve survival or reduce HF hospitalisations for the latter group, which will form the focus of this review.

\section{Epidemiology}

Population studies and registries from around the world have suggested that $30-75 \%$ of the heart failure population have HFpEF. The large variation is related to the differing definitions

Authors: ${ }^{A}$ Department of Cardiology, Royal Brompton Hospital, London, UK; ${ }^{B}$ Department of Cardiology, Baker Heart and Diabetes Institute, Melbourne, Australia; ' Department of Cardiology, Royal Brompton Hospital and Imperial College London, London, UK of HFpEF employed. A recent study from the UK suggested that approximately $20 \%$ of patients hospitalised with HF have a LVEF $\geq 50 \%^{3}$

HFpEF has been suggested to be more common in older patients, females and those with greater comorbidities. The most prevalent comorbidities in HFpEF are hypertension, ischaemic heart disease, diabetes and atrial fibrillation.

Longitudinal follow-up studies have suggested that the prevalence of HFpEF is increasing and may reflect the ageing and greater comorbid population in the developed world as well as an increased awareness of the diagnosis.

\section{Prognosis}

Estimates of mortality rates of patients with HFpEF have varied widely in the literature but, regardless of the exact figure, they have a poorer prognosis than patients with similar comorbidities without HF. Reported 5-year survival from epidemiological studies in patients with $\mathrm{HFpEF}$ is as low as $65 \%$, which is similar to the survival of patients with HFrEF.

\section{Pathophysiology underpinning HFpEF}

HFpEF represents a collection of heterogeneous conditions (Box 1) that can sufficiently elevate left atrial pressures and precipitate clinical features of $\mathrm{HF}$, in the context of a LVEF $\geq 50 \%$. There are several common features, but the growing scientific study of this patient population has shown that they are not one unified group. Previously called 'diastolic heart failure', it is now recognised that this term is inaccurate as abnormalities in HFpEF are not limited to diastolic function (Fig 1). ${ }^{4}$

Previously HFpEF was considered a phenotype that emerged following progressive pressure overload and secondary concentric ventricular hypertrophy that induced diastolic dysfunction and myocardial remodelling. While this mechanism may have explained diastolic dysfunction in patients with HFpEF and coexisting hypertension, the existence of a substantial population of normotensive patients challenged this theory. Consequently, a contemporary theory has emerged positing that myocardial stiffness can result from several different pathophysiological mechanisms, including microvascular inflammation, interstitial fibrosis, disruption to the extracellular matrix, altered cardiomyocyte intracellular calcium cycling and altered myofilament biochemical properties increasing stiffness. Some of these are driven by common HFpEF comorbidities such as hypertension, obesity and diabetes mellitus. Downstream molecular sequelae triggered by microvascular inflammation, results in increased oxidative stress, chronotropic incompetence 


\begin{tabular}{l} 
Box 1. A non-exhaustive list of possible HFpEF \\
aetiologies \\
$>$ Elderly - ageing \\
$>$ Chronic obstructive pulmonary disease \\
$>$ Chronic renal failure \\
$>$ Diabetes \\
$>$ Obesity \\
$>$ Obstructive sleep apnoea \\
$>$ Previous cancer therapy \\
$>$ Chemotherapy \\
$>$ Doxorubicin \\
$>$ Epirubicin \\
$>$ Radiotherapy \\
$>$ Left breast radiotherapy \\
$>$ Post myocardial infarction \\
$>$ Post AVR for severe aortic stenosis \\
$>$ Cardiac amyloidosis \\
$>$ Hypertrophic cardiomyopathy \\
$>$ Reverse remodelled HFrEF \\
\hline AVR
\end{tabular}

$\mathrm{AVR}=$ aortic valve replacement; $\mathrm{HFpEF}=$ Heart failure with preserved ejection fraction; $\mathrm{HFrEF}=$ heart failure with reduced ejection fraction

and insufficient vasodilator reserve. This process consequently leads to the development of interstitial fibrosis and myocardial remodelling, ultimately contributing towards impaired ventricular relaxation and diastolic dysfunction (Fig 2). However, the degree to which a specific process underlies the diastolic impairment in a particular patient remains challenging to identify using current clinical diagnostic techniques.

\section{Diagnosing HFpEF}

Making a diagnosis of HFpEF is not a straightforward matter as it is generally a diagnosis of exclusion, with international guidelines recommending the exclusion of patients with non-cardiac causes of symptoms (eg obesity, lung disease, anaemia). In practice, noncardiac causes of symptoms often coexist in patients with HFpEF and may even be a driving factor of the HF process, which adds to the difficulty in making a diagnosis. The 2016 European Society

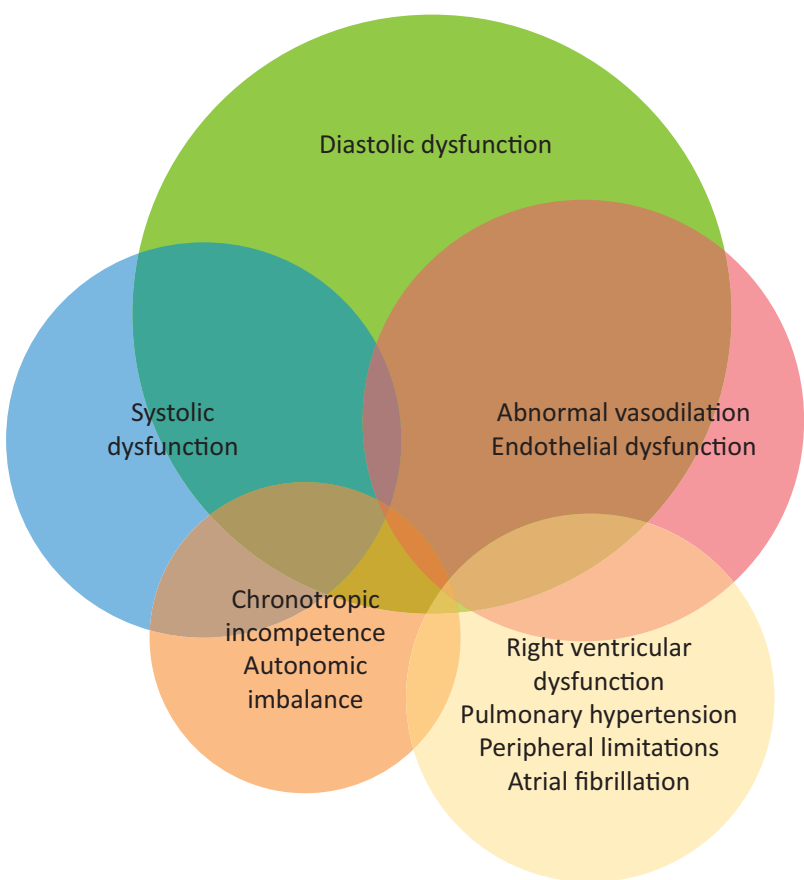

Fig 1. Multiple cardiovascular abnormalities observed in patients with heart failure with preserved ejection fraction. Reproduced with permission from Borlaug (2013). ${ }^{4}$

of Cardiology (ESC) guideline mandates symptoms and signs of heart failure: a non-dilated left ventricle with an LVEF $\geq 50 \%$, along with some evidence of impaired diastolic function, which could include an elevated natriuretic peptide level, left atrial dilatation, left ventricular hypertrophy and/or a minimum of two Doppler echocardiographic features of diastolic dysfunction (Box 2).

Patients with HFpEF tend to have symptoms of HF on exertion. It is well accepted that these patients may not offer objective evidence of HF at rest, but this might be uncovered on exertion ie by undertaking a diastolic stress test, either with an exercise echocardiogram or an exercise right heart catheterisation.

\section{Additional abnormalities of cardiac function in HFpEF}

Beyond diastolic dysfunction, there are additional physiological disruptions that can be detected in patients with HFpEF

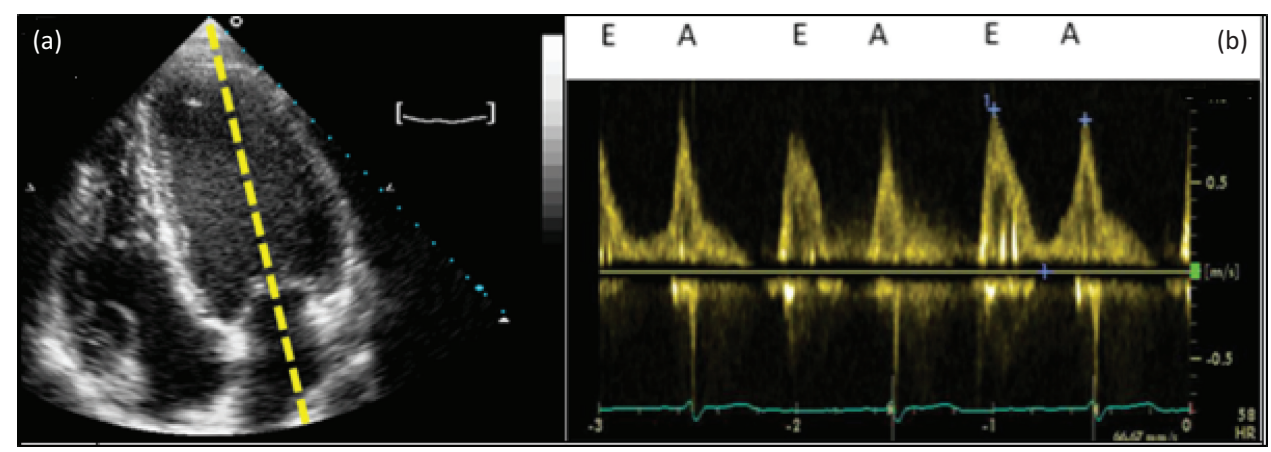

Fig 2. Echocardiographic assessment of diastolic dysfunction. (a) Demonstration of how pulse wave Doppler is measured at the tips of the mitral valve in the left ventricle; (b) measuring the flow of blood from the left atrium into the left ventricle across the mitral valve using pulse wave Doppler enables characterisation of the E wave (representing early diastolic filling) and the A wave (atrial contraction). For a detailed review of diastolic physiology please refer to Mitter et al, 2017. 
Box 2. Quick guide to diagnosing HFpEF based on European Society of Cardiology Guidelines ${ }^{4}$

Signs and symptoms of heart failure

Elevated natriuretic peptide levels (BNP $\geq 35 \mathrm{pg} / \mathrm{mL}$ or NT-proBNP level $\geq 125 \mathrm{pg} / \mathrm{mL}$ )

A preserved ejection fraction ( $L V E F \geq 50 \%$ )

Additional perturbations to cardiac structure and/or function

$>$ left atrial volume index $>34 \mathrm{~mL} / \mathrm{m}^{2}$

$>$ left ventricular mass index $\geq 115 \mathrm{~g} / \mathrm{m}^{2}$ for males and $\geq 95 \mathrm{~g} / \mathrm{m}^{2}$ for females

E to $E^{\prime}$ ratio $\geq 13$

mean E' septal and lateral wall $<9 \mathrm{~cm} / \mathrm{s}$

Stress testing or invasive measurement of elevated LV filling pressures can be performed. There is diagnostic doubt regarding HFpEF.

$\mathrm{BNP}=$ brain natriuretic peptide NT-proBNP; HFpEF = heart failure with preserved ejection fraction; $\mathrm{LVEF}=$ left ventricular ejection fraction; NT-proBNP = N terminal peptide fragment of proBNP

(Fig 1). This includes cardiac features - such as chronotropic incompetence, tachyarrhythmias and intolerance to high heart rates, pulmonary hypertension with right ventricular dysfunction and non-cardiac features. Chronotropic incompetence is frequently detected and is a common limiting factor to a patient's exercise capacity. Pulmonary hypertension and right ventricular dysfunction is also relatively common, occurring in approximately a third of patients. Right ventricular dysfunction is usually the result of elevated pulmonary vein pressures and increased pulmonary artery resistance, consequently resulting in pulmonary hypertension. In some scenarios, right ventricular dysfunction may result from the underlying disease causing left ventricular diastolic impairment, eg cardiac amyloidosis. When present, right ventricular dysfunction is considered a poor prognostic indicator.

\section{Current treatment options for HFpEF}

Unlike HFrEF, there are currently no disease-modifying agents available for HFpEF that improve clinical outcomes. Several randomised controlled trials evaluating the efficacy of therapies routinely administered to patients with HFrEF have failed to demonstrate benefit for HFpEF patients. As such, patients diagnosed with $\mathrm{HFpEF}$ have relatively few treatment options, with ESC guidelines advising the treatment of any underlying comorbidities and resolution of congestion with diuretics when present. ${ }^{6}$

\section{Previous HFpEF trials}

Trials evaluating the efficacy of therapeutic agents routinely used within an HFrEF population have all failed to demonstrate benefit for patients with HFpEF (Fig 3). Consequently, extensive debate surrounding the reasons underpinning lack of efficacy has ensued. There are many reasons these previous trials may have failed, including suboptimal trial design and inadequate attenuation of an intended pathway. However, while many of the primary endpoints for randomised controlled trials investigating HFpEF produced a neutral outcome, there were several secondary outcome measures that may suggest an improvement in outcomes for patients with HFpEF. This includes the TOPCAT (Treatment of Preserved Cardiac Function Heart Failure with an Aldosterone Antagonist)

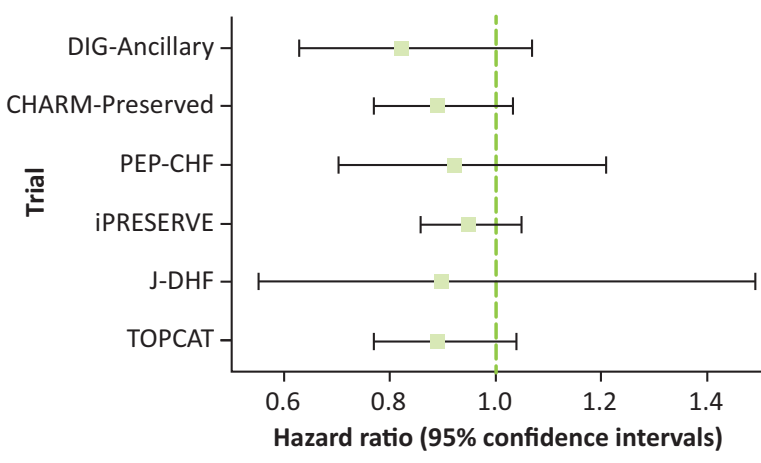

Fig 3. Forest plot summary of the hazard ratios, with $95 \%$ confidence intervals, of the primary endpoint from heart failure with preserved ejection fraction outcome trials.

trial. ${ }^{7}$ Randomising 3,445 participants to receive either a low dose of spironolactone (target dose $30 \mathrm{mg}$ once daily) or placebo, TOPCAT demonstrated a neutral outcome for a composite primary endpoint of cardiovascular mortality, aborted cardiac arrest or HF hospitalisation. ${ }^{7}$ However, there was evidence to suggest that low-dose spironolactone was capable of reducing HF-associated hospitalisations within the spironolactone group (spironolactone $12.0 \%$ versus placebo $14.2 \%$; hazard ratio $[\mathrm{HR}]=0.83(95 \%$ confidence interval [CI] 0.69-0.99; $p=0.04) .{ }^{7}$ However, the patients enrolled within the TOPCAT trial were highly heterogeneous, with a third of patients failing to demonstrate echocardiographic diastolic dysfunction, ${ }^{8}$ and highly variable event rates observed between the American and Russian centres. ${ }^{9}$ Furthermore, a retrospective analysis of serum collected from TOPCAT participants at 12 months has demonstrated higher rates of undetectable levels of canrenone, spironolactone's active metabolite, within individuals recruited to a Russian centre compared with those recruited to American centres (30\% versus $3 \%$; $p<0.001)^{10}$

Therefore, there is a possibility that spironolactone might be beneficial for HFpEF patients, as subgroup analysis of TOPCAT participants with raised BNP levels achieved the primary composite endpoint of death from cardiovascular causes, aborted cardiac arrest or hospitalisation for HF (spironolactone 15.9\% versus placebo $23.6 \%$, HR 0.65, 95\% CI 0.49-0.87; $p=0.003)^{7}$ Additional phase III trials for HFpEF have evaluated the role of therapeutic agents that had previously demonstrated benefit for patients with $\mathrm{HFrEF}$, although these trials also failed to achieve the primary endpoint. ${ }^{11-16}$ This included the evaluation of perindopril within the PEP-CHF (the perindopril in elderly people with chronic heart failure) trial that, despite a neutral primary endpoint outcome, demonstrated benefit with respect to $\mathrm{HF}$ hospitalisation (HR 0.628, 95\% CI 0.408-0.966; $p=0.033$ ), improvement in functional New York Heart Association class $(p<0.030)$ and 6-minute corridor walk distance $(\mathrm{p}=0.011) .{ }^{11}$

Trials have also been conducted to assess therapeutic strategies based on successful preclinical studies. This included sildenafil, a phosphodiesterase- 5 inhibitor, and vericiguat, a soluble guanylate cyclase stimulator. Administration of sildenafil to patients diagnosed with HFpEF was evaluated within the RELAX (Phosphodiesterase-5 Inhibition to Improve Clinical Status and Exercise Capacity in Diastolic Heart Failure) trial. It had been hypothesised that sildenafil would be useful in the treatment of patients with HFpEF and pulmonary hypertension; however, the 
RELAX trial failed to improve clinical status or exercise capacity in patients with HFpEF. ${ }^{17}$ Similarly, vericiguat failed to demonstrate efficacy when evaluated within the prospective, randomised, placebo-controlled double-blind, phase IIb SOCRATES-PRESERVED (SOluble guanylate Cyclase stimulatoR in heArT failurE patientS with PRESERVED EF) study. ${ }^{18}$ While vericiguat was unable to demonstrate a reduction in either NT-proBNP or left atrial volume at 12 weeks, there was evidence to suggest improvements in quality of life.

In addition to pharmacological therapy, investigators have trialled numerous interventional procedures within the HFpEF population, including implantation of an interatrial shunt device and renal sympathetic denervation. Evaluation of the safety and efficacy of an interatrial shunt device was considered within the REDUCE LAP-HF (a transcatheter intracardiac shunt device for heart failure with preserved ejection fraction) trial that was conducted across 21 centres throughout Europe and Australasia. ${ }^{19}$ Hypothesised to reduce left atrial pressures within HFpEF, this phase I, single-arm, open-label study was performed in 64 symptomatic patients diagnosed with HFpEF in whom elevated pulmonary capillary wedge pressures, either at rest $(>15 \mathrm{mmHg})$ or during exercise (>25 mmHg), were detected. Overall, REDUCE LAP-HF provided evidence that suggested implantation of an interatrial shunt device was both a safe and effective method to facilitate left atrial decompression in HFpEF patients who have evidence of raised pulmonary capillary wedge pressures. ${ }^{19,20}$ Consequently, a randomised, placebo-controlled, study (REDUCE LAP HF-I [NCT02600234, ClinicalTrials.gov]) is being pursued to further quantify potential efficacy of interatrial shunt therapy. ${ }^{21}$

Additional interventions have also been pursued, including renal sympathetic denervation. We studied the effect of renal sympathetic denervation (RSD) in patients with HFpEF in a single centre, open-label phase II clinical trial. ${ }^{22}$ This was based on evidence of reverse remodelling of left ventricular hypertrophy (LVH) and LV diastolic stiffness, hallmarks of HFpEF, in patients with hypertensive heart disease treated with RSD. We recruited patients from nine UK hospitals and while we identified that approximately $22 \%$ of 5,883 patients hospitalised with HF at the nine hospitals has a recorded LVEF $\geq 50 \%$ (the large inpatient HFpEF population), it transpired that over $90 \%$ were ineligible for our study for one of four main reasons: extreme age ( $>85$ years), renal impairment (eGFR $<45 \mathrm{~mL} / \mathrm{min}$ ), chronic obstructive pulmonary disease or structural heart diseases, such as hypertrophic cardiomyopathy, severe aortic stenosis and cardiac amyloidosis. Only 45 patients met the inclusion and exclusion criteria, and the ESC definition of HFpEF, before being approached for enrolment. This experience has been replicated elsewhere, with most HFpEF studies failing to recruit on time. Ultimately, 25 patients enrolled and were randomised 2:1 to RSD versus ongoing medical therapy. There was no significant difference in the primary endpoint of a range of relevant structural, functional and symptomatic measurements of $\mathrm{HF}$, and in various exploratory measurements of sympathetic activity. ${ }^{22}$ Current trials are evaluating the efficacy and safety of sacubitril-valsartan (Entresto ${ }^{\text {TM }}$ ) in the PARAGON-HF trial (NCT01920711, ClinicalTrials. gov) and the SGLT-2 inhibitor empagliflozin.

\section{Preventing HFpEF}

Although a broad range of comorbidities can contribute towards $\mathrm{HFpEF}$, most frequently patients with HFpEF are older and have a concomitant diagnosis of hypertension and/or type 2 diabetes mellitus. Both hypertension and type 2 diabetes mellitus can be controlled using agents that reduce cardiovascular events.

\section{Elderly patients with hypertension}

In older patients with hypertension, evidence derived from the HYVET (HYpertension in the Very Elderly Trial) study group has demonstrated that indapamide, in addition to an angiotensinconverting enzyme (ACE) inhibitor (such as perindopril), can reduce the incidence of future $\mathrm{HF}$ (indapamide \pm perindopril 5.3 versus placebo 14.8/1,000 person years; HR 0.36, $95 \%$ CI $0.22-0.58 ; p<0.001)^{23,24}$

\section{Diabetes mellitus}

Until recently, metformin was the only treatment for patients with type 2 diabetes mellitus that improved cardiovascular outcomes. ${ }^{25}$ However, empagliflozin - a sodium-glucose cotransporter-2 (SGLT2) inhibitor - has demonstrated cardiovascular benefit in patients with type 2 diabetes and elevated cardiovascular risk, and specifically reduced the development of $\mathrm{HF}^{26}$ While not clear whether this was prevention of HFpEF or $\mathrm{HFrEF}$, the demographic of this population would be at high risk for HFpEF. Although evidence is derived from a safety analysis study, there was evidence that empagliflozin could potentially reduce new onset $\mathrm{HF}$, HF-associated hospitalisations and cardiovascular-associated mortality in a safety analysis study. ${ }^{26}$ Subgroup analysis of this study revealed a possible signal that patients with established HF (including individuals with HFpEF) may also benefit from empagliflozin, with prospective trials including the phase III EMPEROR-Preserved (EMPagliflozin outcomE tRial in Patients With chrOnic heaRt Failure With Preserved Ejection Fraction) trial (NCT03057951, ClinicalTrials. gov) - underway.

\section{Practical guide to the management of patients with symptomatic HFpEF}

A pragmatic approach should be taken when managing patients with symptomatic HFpEF, while acknowledging the available evidence base. Appreciating that the HFpEF population is heterogeneous, it is important to thoroughly investigate patients with this clinical syndrome to exclude potentially reversible phenotypes, such as ischaemia, uncontrolled hypertension, tachyarrhythmias and chronotropic incompetence. This can be achieved using Holter monitors, non-invasive blood pressure monitoring, urinalysis, myocardial perfusion scans and physiological stress echocardiography. Holter monitoring is effective for the detection of heart rate responsiveness, including the presence of atrial fibrillation and its rate control, inappropriate sinus tachycardia and chronotropic incompetence. Non-invasive blood pressure monitoring is useful to ensure satisfactory blood pressure control and urinalysis helps identify proteinuria or nephrotic syndrome. Myocardial perfusion scans are useful for the detection of ischaemic heart disease, particularly in older patients. When a diagnosis is still uncertain, based on resting imaging, physiological stress echocardiography can demonstrate clinical utility.

If there is evidence of inducible ischaemia, revascularisation options should be discussed with the patient. Similarly, if 
chronotropic incompetence is identified, pacemaker implantation may be necessary to allow patients to appropriately raise their heart rate with exercise.

For patients with hypertension, the HYVET trial demonstrated that, irrespective of age, the introduction of an ACE inhibitor was effective. However, if ACE inhibitors are not tolerated, the LIFE (Losartan Intervention for Endpoint Reduction in Hypertension) trial supports introduction of an angiotensin-receptor blocker, such as losartan. $^{22}$

Diuretic therapy is also advisable for patients with hypertension and/or oedema. Individuals over the age of 80 years benefit most from indapamide, whereas ACE inhibitors should be considered for younger patients. Based on the TOPCAT subgroup analysis, introduction of low-dose spironolactone in HFpEF patients with elevated BNP or NT-proBNP levels is an option with close monitoring of renal function and potassium. For patients with type 2 diabetes, initial reports suggest the use of an SGLT2 inhibitor, such as empagliflozin, is effective at managing cardiovascular risk factors.

It is advised that patients with $\mathrm{HFpEF}$ are managed by specialists with an interest in HF. With over 60 clinical trials of HFpEF globally, many specialist centres across UK, including the Royal Brompton Hospital, are participating in research to help meet this unmet clinical need.

\section{Conclusions}

HFpEF does not represent a single condition, and is instead an eclectic compilation of aetiologies that contribute towards a disruption in diastolic functioning. While there are many ongoing clinical trials, there are no disease-modifying agents available for the treatment of HFpEF. Instead, clinicians should thoroughly look for an underlying aetiology and target any potentially reversible causes. Furthermore, rather than attempting to unearth a single 'magic bullet' treatment that may improve the outcomes of all HFpEF patients, future trials should instead be pragmatic and focus on evaluating specific, highly phenotyped, HFpEF subsets that may help advance the treatment options available to a proportion of this largely underserved population.

\section{Conflicts of interest}

The authors have no conflicts of interest to declare.

\section{References}

1 National Institute for Health and Care Excellence. Chronic heart failure in adults: management. NICE clinical guideline No 108. London: NICE, 2010.

2 Juenger J, Schellberg D, Kraemer S et al. Health related quality of life in patients with congestive heart failure: comparison with other chronic diseases and relation to functional variables. Heart 2002:87:235-41.

3 Patel HC, Hayward C, di Mario C et al. Heart failure with preserved ejection fraction: the impact of stricter definitions. Eur J Heart Fail 2014:16:767-71

4 Borlaug BA. The pathophysiology of heart failure with preserved ejection fraction. Nat Rev Cardiol 2014:11:507-15.

5 Mitter SS, Shah SJ, Thomas JD. A test in context: E/A and E/e' to assess diastolic dysfunction and LV filling pressure. J Am Coll Cardiol 2017;69:1451-64
6 Ponikowski P, Voors AA, Anker SD et al. 2016 ESC Guidelines for the diagnosis and treatment of acute and chronic heart failure: The Task Force for the diagnosis and treatment of acute and chronic heart failure of the European Society of Cardiology (ESC). Developed with the special contribution of the Heart Failure Association (HFA) of the ESC. Eur ] Heart Fail 2016:18:891-975.

7 Pitt B, Pfeffer MA, Assmann SF et al. Spironolactone for heart failure with preserved ejection fraction. N Engl ] Med 2014;370:1383-92.

8 Shah AM, Shah S], Anand IS et al. Cardiac structure and function in heart failure with preserved ejection fraction: baseline findings from the echocardiographic study of the treatment of preserved cardiac function heart failure with an aldosterone antagonist trial. Circ Heart Fail 2014;7:104-15.

9 Pfeffer MA, Claggett B, Assmann SF et al. Regional variation in patients and outcomes in the treatment of preserved cardiac function heart failure with an aldosterone antagonist (TOPCAT) Trial. Circulation 2015;131:23-42.

10 de Denus S, O'Meara E, Desai AS et al. Spironolactone metabolites in TOPCAT - new insights into regional variation. $N$ Engl J Med 2017:376:1690-2.

11 Cleland JGF, Tendera M, Adamus ] et al. The perindopril in elderly people with chronic heart failure (PEP-CHF) study. Eur Heart ] 2006;27:2338-45

12 Ahmed A, Rich MW, Fleg JL et al. Effects of digoxin on morbidity and mortality in diastolic heart failure: the ancillary digitalis investigation group trial. Circulation 2006;114:397-403.

13 Yusuf S, Pfeffer MA, Swedberg K et al. Effects of candesartan in patients with chronic heart failure and preserved leftventricular ejection fraction: the CHARM-Preserved Trial. Lancet 2003;362:777-81.

14 Massie BM, Carson PE, McMurray J] et al. Irbesartan in patients with heart failure and preserved ejection fraction. N Engl J Med 2008;359:2456-67.

15 Edelmann F, Wachter R, Schmidt AG et al. Effect of spironolactone on diastolic function and exercise capacity in patients with heart failure with preserved ejection fraction: the Aldo-DHF randomized controlled trial. JAMA 2013;309:781-91.

16 Pal N, Sivaswamy N, Mahmod M et al. Effect of selective heart rate slowing in heart failure with preserved ejection fraction. Circulation 2015;132:1719-25.

17 Redfield MM, Chen HH, Borlaug BA et al. Effect of phosphodiesterase- 5 inhibition on exercise capacity and clinical status in heart failure with preserved ejection fraction: a randomized clinical trial. JAMA 2013:309:1268-77.

18 Pieske B, Maggioni AP, Lam CSP et al. Vericiguat in patients with worsening chronic heart failure and preserved ejection fraction: results of the SOluble guanylate Cyclase stimulatoR in heArT failurE patientS with PRESERVED EF (SOCRATES-PRESERVED) study. Eur Heart J 2017:38:1119-27.

19 Hasenfuß G, Hayward C, Burkhoff D et al. A transcatheter intracardiac shunt device for heart failure with preserved ejection fraction (REDUCE LAP-HF): a multicentre, open-label, single-arm, phase 1 trial. Lancet 2016;387:1298-304.

20 Kaye DM, Hasenfuß G, Neuzil P et al. One-year outcomes after transcatheter insertion of an interatrial shunt device for the management of heart failure with preserved ejection fraction. Circ Heart Fail 2016;9:e003662.

21 Feldman T, Komtebedde J, Burkhoff D et al. Transcatheter interatrial shunt device for the treatment of heart failure: rationale and design of the randomized trial to REDUCE Elevated Left Atrial Pressure in Heart Failure (REDUCE LAP-HF I). Circ Heart Fail 2016;9:003025

22 Patel HC, Rosen SD, Hayward C et al. Renal denervation in heart failure with preserved ejection fraction (RDT-PEF): a randomized controlled trial. Eur J Heart Fail 2016;18:703-12. 
23 Beckett NS, Peters R, Fletcher AE et al. Treatment of hypertension in patients 80 years of age or older. N Engl J Med 2008;358:1887-98.

24 van Vark LC, Bertrand M, Akkerhuis KM et al. Angiotensin-converting enzyme inhibitors reduce mortality in hypertension: a meta-analysis of randomized clinical trials of renin-angiotensin-aldosterone system inhibitors involving 158,998 patients. Eur Heart ] 2012;33:2088-97.

25 UK Prospective Diabetes Study (UKPDS) Group. Effect of intensive blood-glucose control with metformin on complications in overweight patients with type 2 diabetes (UKPDS 34). Lancet 1998;352:854-65.
26 Zinman B, Wanner C, Lachin JM et al. Empagliflozin, cardiovascular outcomes, and mortality in type 2 diabetes. N Engl J Med 2015:373:2117-28

Address for correspondence: Dr Alexander R Lyon, Heart Failure Service, Department of Cardiology, Royal Brompton Hospital, London SW3 6NP, UK.

Email: A.Lyon@rbht.nhs.uk 